\title{
DIRETRIZES PARA AVALIAÇÃO DE SISTEMAS DE ORGANIZAÇÃO DO CONHECIMENTO REPRESENTADOS EM SKOS
}

\section{GUIDELINES FOR EVALUATION OF KNOWLEDGE ORGANIZATION SYSTEMS REPRESENTED IN SKOS}

\author{
Rogério Aparecido Sá Ramalhoa \\ Janailton Lopes Sousab
}

\begin{abstract}
RESUMO
Introdução: O Modelo de Dados Simple Knowledge Organization System (SKOS) é uma recomendação do World Wide Web Consortium (W3C) para a representação de Sistemas de Organização do Conhecimento em ambientes digitais, na última década o modelo SKOS tem contribuído para um aumento exponencial da quantidade de vocabulários publicados em ambientes digitais, evidenciando a necessidade de estudos que favoreçam uma melhor avaliação destes vocabulários. Objetivo: Apresentar uma sistematização de diretrizes para a avaliação de vocabulários publicados em SKOS. Metodologia: Pesquisa exploratória de caráter bibliográfica e documental, com abordagem teórica e qualitativa. Resultados: É apresentada uma sistematização de estudos que possam contribuir para a sistematização de diretrizes para a avaliação de vocabulários codificados em SKOS. Conclusões: A representação de vocabulários controlados em ambientes digitais incorpora inúmeras potencialidades aos Sistemas de Organização do Conhecimento, porém também apresenta novos desafios, principalmente no que se refere à avaliação de requisitos técnicos e definição formal dos relacionamentos, evidenciando a importância de estudos que contribuam para um maior aperfeiçoamento dos processos de avaliação de vocabulários em ambientes digitais.
\end{abstract}

Descritores: Sistemas de Organização do Conhecimento. Vocabulários Controlados. Avaliação. Diretrizes. SKOS.

\section{INTRODUÇÃO}

O Simple Knowledge Organization System (SKOS) favorece uma aproximação entre os Sistemas de Organização do Conhecimento tradicionais

\footnotetext{
a Professor do Programa de Pós-Graduação em Ciência da Informação da Universidade Federal de São Carlos (PPGCI-UFSCar). E-mail: ramalho@ufscar.br

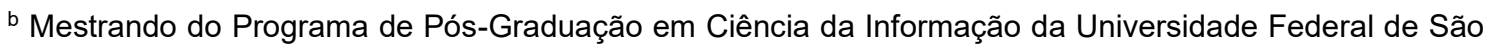
Carlos (PPGCI-UFScar). E-mail: emailjanailton@trocar.br
} 
com as novas potencialidades oferecidas pelas tecnologias semânticas, desenvolvido com base no Resource Description Framework (RDF) e eXtensible Markup Language (XML), favorece novas potencialidades para a representação de vocabulários em ambientes digitais (RAMALHO, MARTINS; SOUSA, 2017).

Conforme relatam Pastor-Sanchez, Martínez-Mendez e Rodriguez-Muñoz (2012) e Miles e Bechhofer (2009) o desenvolvimento do padrão SKOS teve início no ano de 2002, mas apenas em 2009 passou a ser considerando como uma recomendação oficial do World Wide Web Consortium (W3C) para a representação de Knowledge Organization Systems (KOS) na Web.

Broughton (2010) destaca a proximidade do SKOS com as classificações facetadas, ressaltando que a teoria da classificação facetada pode fornecer um modelo para os elementos utilizados nos sistemas de representação baseados em tecnologias semânticas, bem como terminologias para habilitá-los. Neste sentido, Mustafa El Hadi (2015) afirma que o padrão SKOS inaugura uma nova etapa de sistemas de representação, capazes de proporcionar uma dimensão semântica praticamente inexplorada em ambientes digitais.

De acordo com Isaac e Summer (2009) o padrão SKOS possibilita que conceitos possam ser identificados utilizando Uniform Resource Identifier (URIs), etiquetados a partir de diferentes tipos de anotações, estruturando hierarquias e redes associativas que possibilitam relacionar diferentes esquemas conceituais.

Os KOS são agrupamentos com preditivas lógicas e semânticas aplicadas a recuperação da informação, no âmbito tecnológico, os vocabulários controlados tornaram-se a grande matéria prima para manipulação desses sistemas na web por meios dos dados que referenciam os objetos. A utilização de vocabulários interligados favorece a aquisição de conhecimento, possibilitando contextualizar formalmente os relacionamentos existentes entre os dados (MÉNDEZ; GREENBERG, 2012).

O conceito é o elemento chave do SKOS, fornecendo uma estrutura simplificada, porém flexível, capaz de incorporar novas potencialidades de representação (RAMALHO, 2015).

De acordo com a Teoria do Conceito apresentada por Dahlberg (1978) os conceitos possuem relações lógicas, hierárquicas, partitivas, de oposição e 
funcionais em KOS. Gomes (1990) complementam que um autêntico tesauro é construído a partir dos conceitos, apresentados num arranjo sistemático.

Pastor-Sanchez, Martinez-Rodriguez e Mendez-Muñoz (2009), afirmam que a exploração de vocabulários na Web ainda é muito limitada, destacando que o próprio conceito de tesauro tem evoluído para adaptar-se aos novos modelos de representação da Web, abandonando o paradigma lexical em favor de um paradigma conceitual. Nesta perspectiva, o modelo SKOS fornece um modelo para expressar a estrutura básica e o conteúdo de vocabulários em ambientes digitais (ISAAC; SUMMERS, 2009).

Assim, considerando o aumento exponencial da quantidade de vocabulários publicados em ambientes digitais, apresenta-se como objetivo a identificação de diretrizes para a avaliação de vocabulários publicados em SKOS. O presente trabalho está vinculado a um projeto de pesquisa em andamento, financiado pelo CNPq e FAPESP e fundamenta-se em uma pesquisa exploratória de caráter bibliográfico/documental, com abordagem teórica e qualitativa.

\section{VOCABULÁRIOS CONTROLADOS EM SKOS}

Vocabulários controlados são caracterizados como formas sistêmicas de representar informações, onde é possível aplicar diversos tipos de relacionamentos lógicos e semânticos. Representar uma linguagem natural a partir da utilização de uma linguagem artificial/controlada é um processo complexo, de modo que historicamente áreas como a Terminologia e a Ciência da Informação tem desenvolvido subsídios teóricos que favoreçam o aperfeiçoamento dos processos de elaboração de vocabulários controlados.

Ao longo da última década o modelo SKOS tem se destacado como uma alternativa viável para a representação de vocabulários em ambientes digitais, favorecendo a interoperabilidade e a manutenção da integridade dos elementos que compõem esquemas conceituas. A identificação de um vocabulário SKOS consiste em verificar a existência de instâncias diretas do tipo <skos:Concept>; e instâncias implícitas devido ao domínio restrições de alcance nas relações 
SKOS (MANAF; BECHHOFER; STEVENS, 2012).

O modelo SKOS possibilita a representação de diferentes tipos de relações semânticas entre conceitos, a partir da utilização do SKOS Core Vocabulary, que é um conjunto de propriedades e classes utilizadas para expressar o conteúdo e estrutura de um esquema de conceitos em RDF (CATARINO; CERVANTES; ANDRADE, 2015).

Segundo Isaac e Summers (2009) SKOS fornece um modelo para expressar a estrutura básica e o conteúdo de vocabulários controlados, de modo que um conceito possa ser formalmente associado a um termo e relacionado com outros conceitos, espelhando as categorias fundamentais de relações usadas em vocabulários.

Atualmente existem diversos vocabulários controlados internacionais amplamente utilizados representados em SKOS, podendo-se destacar: Thesaurus multilingual AGROVOC; General Multilingual Environmental Thesaurus (GEMET); Multilingual Thesaurus of the European Union (EuroVoc); STW Thesaurus for Economics; UNESKOS; entre outros.

Em âmbito nacional pode-se destacar iniciativas representação de vocabulários em SKOS como o Vocabulário Controlado de Governo Eletrônico (VCGE) e o Tesauro Brasileiro de Ciência da Informação (TBCl), que passou a ser oficialmente disponibilizado em SKOS a partir do trabalho de Santos, Cervantes e Fujita (2018).

Para a representação de Sistemas de Organização do Conhecimento em SKOS é necessário a formalização das propriedades e relacionamentos existentes entre os conceitos, favorecendo que máquinas possam identificar e processar automaticamente as informações representadas. A figura 01 apresenta graficamente um trecho do TBCl codificado em SKOS. 
Figura 01 - Representação gráfica de um trecho do TBCl em SKOS

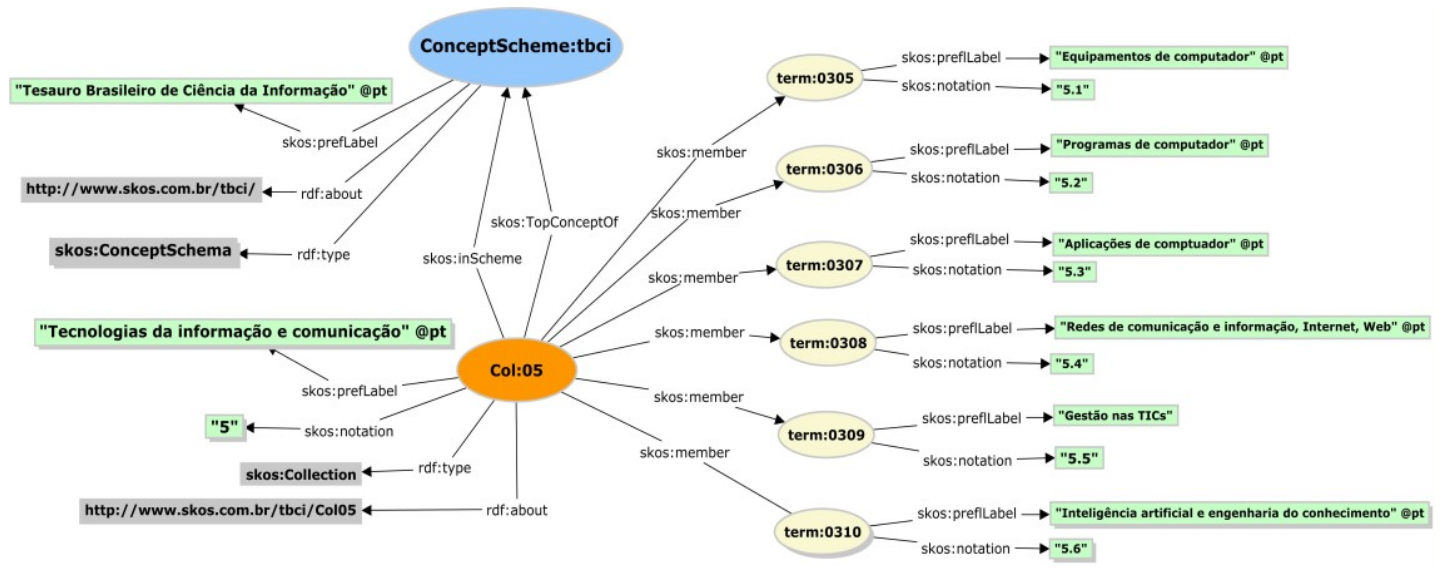

Fonte: Ramalho (2017)

O modelo SKOS favorece a interoperabilidade entre diferentes Esquemas Conceituais, possibilitando identificar as relações existentes entre um Conceito e um determinado Esquema Conceitual, a partir da utilização da propriedade skos:ConceptScheme. Além disso, conforme pode ser observado na figura 01 , todas as propriedades e relacionamentos necessitam ser formalmente representados a partir de linguagens formais.

A representação formal dos elementos que compõem um Sistema de Organização do Conhecimento contribui para o desenvolvimento de práticas profissionais inovadoras e favorece a incorporação de novas potencialidades de utilização de vocabulários em sistemas automatizados. Contudo, tais avanços também trazem no bojo de seu desenvolvimento desafios, principalmente no que se refere ao estabelecimento de diretrizes para a avaliação de vocabulários em ambientes digitais.

\section{DIRETRIZES PARA AVALIAÇÃO DE VOCABULÁRIOS EM SKOS}

Historicamente a área de Ciência da Informação possui uma ampla trajetória no desenvolvimento de diretrizes e métodos de avaliação de vocabulários controlados, fundamentando-se em diferentes tipos de abordagens (objetiva/subjetiva; automática/manual) e direcionadas para as mais variadas dimensões de avaliação. 
De acordo com a norma ANSI/NISO Z39.19 (2010) o número de pesquisas que abordam especificamente a realização de testes e avalição de vocabulários controlados ainda é limitado, evidenciando a necessidade de estudos que abordem esta temática.

De certo modo o número limitado de pesquisas que abordam a avaliação de vocabulários pode ser parcialmente justificado pela dificuldade de se isolar a qualidade de um vocabulário, com o intuito de favorecer avaliações objetivas, a partir do desempenho de outros elementos de um sistema de informação, como tecnologia, mecanismo de pesquisa e interface.

Nesta perspectiva, buscando favorecer a sistematização de diretrizes que possam servir de substrato teórico para a avaliação de vocabulários SKOS foi realizada uma revisão de literatura sobre pesquisas que abordam a avaliação de vocabulários controlados na área de Ciência da Informação. Após o levantamento realizado foram selecionados 10 trabalhos, considerando como critérios de seleção a relevância para a área e o enfoque desta pesquisa, sendo 5 trabalhos que abordam a avaliação de KOS tradicionais e 5 trabalhos direcionados para a avaliação KOS representados em SKOS. Além dos 10 trabalhos, também foram consideradas para esta análise as diretrizes previstas na norma ANSI/NISO (2010).

De acordo com a norma ANSI/NISO (2010) os processos de avaliação de vocabulários controlados podem ser caracterizados em três tipos de avaliações:

- Avaliação heurística - realizada por um perito ou um quórum de especialistas, podendo ser informal e qualitativa ou formal e quantitativa.

- Modelagem de afinidade - reúne uma amostra representativa de usuários para avaliar um vocabulário, solicitando uma classificação dos temos.

- Teste de usabilidade, uma avaliação holística do sistema de informação que pode fornecer informações sobre a eficácia do vocabulário controlado.

Entre as pesquisas com foco na avaliação de KOS tradicionais pode-se destacar o trabalho de Soergel (2002) que apresenta como critérios de avaliação 
a definição de um padrão para denominação de termos; identificação dos tipos de relações entre termos; regras para atualização, entre outros.

Lancaster (2002) aponta algumas medidas para avaliação de vocabulários, tais como: razão de equivalência; razão de reciprocidade; definição; flexibilidade; nível de pré-coordenação e tamanho do agrupamento de termos.

Gil-Leiva (2008) e Monreal (2009) apresentam os conceitos de avaliação intrínseca e extrínseca, sendo a avaliação intrínseca desenvolvida a partir dos resultados da indexação com o fim na qualidade, podendo ser qualitativa ou quantitativa. Em relação a avaliação extrínseca, tem por base a avaliação do resultado da indexação, mediante a interconsistência e recuperação.

Monreal e Gil-Leiva (2011) destacam que a avaliação intrínseca pode ser realizada com o objetivo de analisar os próprios vocabulários controlados favorecendo o estudo da sua estrutura, campos temáticos, notas de escopo, relações semânticas e grau de especificidade.

Em relação a avaliação de vocabulário SKOS é necessário inicialmente verificar se a estrutura e propriedades estão representadas de maneira adequada em relação às recomendações estabelecidas pelo W3C. Nesta perspectiva, as pesquisas de Suominen e Hyvönen (2012); Mader, Haslhofer e Isaac (2012) e Suominen; Mader, (2014) apresentam abordagens automáticas de avaliação, alinhando os fundamentos normativos e as potencialidades oferecidas pela infraestrutura do SKOS.

Suominen e Hyvönen (2012) descrevem alguns critérios para validação de vocabulários SKOS. É possível categorizar estes critérios em "Validações Qualitativas" "Validações Qualitativas", conforme apresentado na Tabela 01. As validações Qualitativas são utilizadas para verificar se determinados elementos, que poderiam inviabilizar a utilização do vocabulário, atendem às recomendações do W3C, tendo como resposta um valor binário (verdadeiro ou falso). As validações Quantitativas têm como objetivo analisar o número de erros existentes em cada um dos critérios avaliados, retornado um valor numérico. 
Tabela 01 - Categorização das validações em SKOS

\begin{tabular}{|l|l|}
\hline \multirow{4}{*}{$\begin{array}{l}\text { Validações } \\
\text { Qualitativas }\end{array}$} & Validação do URI \\
\cline { 2 - 3 } & Etiquetas ausentes \\
\cline { 2 - 3 } & Classes Disjuntas \\
\cline { 2 - 3 } Validações & Consistência de Etiquetas \\
\cline { 2 - 3 } & Consistência de Propriedades de Mapeamento \\
\cline { 2 - 3 } & Consistência das Relações Semânticas \\
\hline \multirow{4}{*}{} & Número de Etiquetas de Idiomas Ausentes \\
\cline { 2 - 3 } & Número de Conceitos Soltos \\
\cline { 2 - 3 } & Número de Valores de Etiquetas ambíguos \\
\cline { 2 - 3 } & Número de sobreposições de propriedades \\
\cline { 2 - 3 } & Número de Relações Semânticas Disjuntas \\
\cline { 2 - 3 } & Número de Ciclos na Hierarquia mais ampla \\
\cline { 2 - 3 } & Número de Espaços em branco extra \\
\hline
\end{tabular}

Fonte: Adaptado de Suominen e Hyvönen (2012)

Além dos estudos de Suominen e Hyvönen (2012), outro trabalho relevante na temática de avaliação de vocabulários SKOS é o artigo de Mader, Haslhofer e Isaac (2012), que classificam os problemas comumente identificados em vocabulários SKOS em 3 categorias: 'Problemas de Rotulagem e Documentação'; 'Problemas Estruturais' e 'Problemas de Vinculação de Dados', conforme apresentado na Tabela 02.

A terceira categoria aplica-se notadamente a problemas de recursos informacionais que podem estar desvinculados dos seus endereços de localização, impossibilitando a vinculação dos dados.

\begin{tabular}{|c|l|}
\hline \multicolumn{2}{|c|}{ Principais tipos de problemas identificados em Vocabulários SKOS } \\
\hline Problemas de & Ausência de Etiquetas de Idioma \\
\cline { 2 - 2 } Rotulagem e & Etiquetas de Idioma invalidas \\
\cline { 2 - 2 } Documentação & Cobertura incompleta de Idiomas \\
\hline
\end{tabular}




\begin{tabular}{|c|c|}
\hline & Conceitos não documentados \\
\hline & Conflitos de etiqueta \\
\hline & Número de Etiquetas de Idiomas Ausentes \\
\hline \multirow{7}{*}{$\begin{array}{l}\text { Problemas } \\
\text { Estruturais }\end{array}$} & Conceitos órfãos \\
\hline & Componentes fracamente conectados \\
\hline & Relações hierárquicas cíclicas \\
\hline & Relações associativas sem valor \\
\hline & Conceitos únicos transitivamente relacionados \\
\hline & Conceitos superiores omitidos \\
\hline & Conceito superior com conceitos mais amplos. \\
\hline \multirow{3}{*}{$\begin{array}{l}\text { Problemas de } \\
\text { Vinculação de } \\
\text { Dados }\end{array}$} & Links ausentes \\
\hline & Links quebrados \\
\hline & Recursos SKOS indefinidos \\
\hline
\end{tabular}

Fonte: Adaptado de Mader, Haslhofer e Isaac (2012)

Diante da revisão de literatura realizada constatou-se que, complementando as diretrizes e pesquisas que abordam a avaliação de KOS tradicionais, as pesquisas de Suominen e Hyvönen (2012); Mader, Haslhofer e Isaac (2012); Suominen e Mader, (2014) fornecem subsídios para identificação e sistematização de diretrizes que possibilitam a avaliação de um vocabulário SKOS.

\section{CONSIDERAÇÕES FINAIS}

O aumento exponencial da quantidade de vocabulários publicados em ambientes digitais representa um avanço significativo para área de Ciência da Informação, apresentando novas possibilidades de atuação e contribuindo para uma maior interoperabilidade semântica entre sistemas.

Nesta perspectiva, torna-se essencial o estabelecimento de diretrizes e métodos de avaliação no intuito de garantir a qualidade dos vocabulários disponibilizados em ambientes digitais, contribuindo para a potencialização dos processos de recuperação a partir da representação de dados vinculados. 
No que tange a representação de vocabulários em SKOS problemas de etiquetagem, estruturação e vinculação dos dados muitas vezes são acarretados por inconsistências decorrentes de processo de inserção automática ou conversão de vocabulários concebidos originalmente de maneira tradicional, além de possíveis erros de tradução, links quebrados e informações incompletas inseridas manualmente.

Uma avaliação que consiga detectar esses tipos de erros, evidencia sua importância, principalmente quando aplicadas a vocabulários muito extensos, ou que foram convertidos para o padrão SKOS, como uma espécie de validação. A utilização de métricas automáticas auxiliam na objetivação deste processo, pois simplificam os critérios de avaliação orientados pela própria recomendação que especifica o uso do SKOS.

A partir da pesquisa realizada constatou-se que os estudos de Suominen e Hyvönen (2012); Mader, Haslhofer e Isaac (2012); Suominen e Mader, (2014) podem contribuir para uma sistematização de diretriz para avaliação de vocabulários codificados em SKOS, pois a maioria dos problemas identificados em Vocabulários SKOS estão relacionados com a conectividade entre os recursos informacionais e aspectos formais de representação.

A representação de vocabulários controlados em ambientes digitais incorpora inúmeras potencialidades aos Sistemas de Organização do Conhecimento, porém também apresenta novos desafios, principalmente no que se refere à avaliação de requisitos técnicos e definição formal dos relacionamentos, evidenciando a importância de estudos que contribuam para um maior aperfeiçoamento dos processos de avaliação de vocabulários em ambientes digitais.

\section{REFERÊNCIAS}

ANSI/NISO. Z39.19-2005 (R2010): guidelines for the construction, format, and management of monolingual controlled vocabularies. NISO, 2010.

BROUGHTON, V. Concepts and terms in the faceted classification: the case of UDC. Knowledge Organization, v. 37, n. 4, 2010. p. 270-279. 
CATARINO, M. E.; CERVANTES, B. M. N.; ANDRADE, I. A. de. A representação temática no contexto da web semântica. Inf. \& Soc.:Est., João Pessoa, v. 25, n. 3, p. 105-116, set./dez. 2015.

DAHLBERG, I. Teoria do conceito. Ciência da Informação, Rio de Janeiro, v.7, n.2, p.101-107, 1978.

GOMES, H. E. Manual de elaboração de tesauros monolíngues. Brasília: CNPq/PNBU, 1990. 78 p.

GIL-LEIVA, I. Manual de indización: teoría y práctica. Gijón, Spain: Trea, 2008.

ISAAC, A.; SUMMERS, E. (Ed.). SKOS Simple Knowledge Organization System Primer: W3C Working Group Note, 18 August 2009. Disponível em: https://www.w3.org/TR/2009/. Acesso em: 19 jan. 2019.

LANCASTER, F. W. El control del vocabulario en la recuperación de la información. 2 ed. Valencia: Universidad de Valencia, 2002.

MADER, C.; HASLHOFER, B.; ISAAC, A. Finding quality issues in SKOS vocabularies. TPDL, 2012.

MANAF, N. A. A.; BECHHOFER, S.; STEVENS, R. The current state of SKOS vocabularies on the Web. In: SIMPERL, E. et al. (Eds.). Extended Semantic Web Conference - ESWC, 2012. p. 270-284.

MÉNDEZ, E.; GREENBERG, J. Linked data for open vocabularies and HIVE's global framework. El Profesional de la Información, v. 21, n. 3, p. 236-244, 2012.

MILES, A.; BECHHOFER, S. SKOS Simples Knowledge Organization System Reference. W3C Recommendation, 2009. Disponível em: http://www.w3.org/TR/skos- reference/. Acesso em: 20 jan 2019.

MUSTAFA EL HADI, W. Cultural interoperability and knowledge organization Systems. In: GUIMARÃES, J. A. C.; DODEBEI, V. (Orgs.). ORGANIZAÇÃO DO CONHECIMENTO E DIVERSIDADE CULTURAL. 1 ed. Marília: ISKOBrasil; FUNDEPE, v. 1, p. 575-606, 2015.

PASTOR-SANCHEZ, J. A.; MARTINEZ-MENDEZ, F. J.; RODRIGUEZ-MUÑOZ, J.V. Aplicación de SKOS para la interoperabilidad de vocabularios controlados en el entorno de linked open data. El profesional de la información, v. 21, n. 3, p. 245-253, 2012.

PASTOR-SANCHEZ, J. A.; MENDEZ, F. J. M.; RODRÍGUEZ-MUÑOZ, J. V. Advantages of thesaurus representation with the Simple Knowledge Organization System (SKOS) compared with other proposed alternatives. 
Information Research, v. 14, n. 4, dez. 2009. Disponível em: http://www.informationr.net/ir/14- 4/paper422.html. Acesso em 04 jun. 2018.

RAMALHO, R. A. S.; MARTINS, P. G. M.; SOUSA, J. L. Evolução das linguagens de marcação: um breve histórico à luz da área de Ciência da Informação. Inf. Prof., Londrina, v. 6, n. 2, jul./dez. 2017. p. 20 - 34. Disponível em: http://www.uel.br/revistas/uel/index.php/infoprof/article/view/33267. Acesso em 10 jan 2019.

RAMALHO, R. A. S. Análise do modelo de dados SKOS: Sistema de Organização do Conhecimento Simples para a Web. Marília/João Pessoa, Informação \& Tecnologia (Itec), v. 2, n. 1, jan./jul., 2015. p. 66-79.

RAMALHO, R.A.S. Representação SKOS da categoria tecnologia da informação e comunicação do tesauro brasileiro de ciência da informação: um estudo preliminar. Tendências da Pesquisa Brasileira em Ciência da Informação, v. 10, n. 2, 6 jun. 2017. Disponível em: http://ejournal.space/tpbci/index.php/tpbci/issue/view/4. Acesso em: 14 nov. 2019.

SANTOS, J. C. F.; CERVANTES, B. M. N.; FUJITA, M. S. L. Tesauro eletrônico: importação no TemaTres e disponibilização na web. In: ENCONTRO NACIONAL DE PESQUISA EM CIÊNCIA DA INFORMAÇÃO, 19., 2018, Londrina. Anais [...]. Londrina: Uel, 2018. Acesso em: 12 fev. 2019.

SOERGEL, D. Thesauri and ontologies in digital libraries: tutorial. In: Evaluation of thesauri. Joint Conference on Digital Libraries, Portland, Oregon, July 14, 2002. Disponível em: http://www.dsoergel.com/cv/B63.pdf. Acesso em 08 set 2018.

MONREAL, C. S.; GIL-LEIVA, I. Evaluation of controlled vocabularies by interindexer consistency. Information Research: an international electronic journal, v. 16, n. 4, dez. 2011. Disponível em: http://www.informationr.net/ir/164/paper502.html. Acesso em: 10 jan 2019.

MONREAL, M. C. S. Evaluación de vocabularios controlados en la indización de documentos mediante índices de consistencia entre indizadores. Departamento. de Comunicación Audiovisual, Documentación e Historia del Arte (TESIS DOCTORAL). Universitat Politècnica de València. Valencia, 2009.

SUOMINEN, O., MADER, C. Assessing and improving the quality of SKOS vocabularies. Journal Data Semantics, v. 3, n. 1, 2014. p. 47-73.

SUOMINEN, O.; HYVÖNEN, E. Improving the quality of SKOS vocabularies with Skosify. In: INTERNATIONAL CONFERENCE ON KNOWLEDGE ENGINEERING AND KNOWLEDGE MANAGEMENT (EKAW 2012), Anais [...]. Springer-Verlag, Gal-way, Ireland, October, 2012. 


\section{GUIDELINES FOR EVALUATION OF KNOWLEDGE ORGANIZATION SYSTEMS REPRESENTED IN SKOS}

Introduction: The Simple Knowledge Organization (SKOS) is a W3C recommendation designed for representation of Knowledge Organization Systems in digital environments, in the last decade the SKOS model has contributed to an exponential increase in the number of vocabularies published in digital environments, highlighting the need to studies that favor a better evaluation of these vocabularies. Objective: To present a systematization of guidelines for the evaluation of vocabularies published in SKOS. Methodology: Exploratory research of bibliographical and documentary character, with theoretical and qualitative approach. Results: Studies are presented that can contribute to a systematization of guidelines for the evaluation of coded vocabularies in SKOS is presented. Conclusions: The representation of controlled vocabularies in digital environments incorporates many potentialities to Knowledge Organization Systems, but also presents new challenges, especially in relation to the evaluation of technical requirements and formal definition of relationships, highlighting the importance of studies that contribute to a greater refinement of vocabulary evaluation processes in digital environments.

Descriptors: Knowledge Organization Systems. Controlled Vocabularies. Evaluation. Guidelines. SKOS.

\section{DIRECTRICES PARA LA EVALUACIÓN DE SISTEMAS DE ORGANIZACIÓN DEL CONOCIMIENTO REPRESENTADOS EN SKOS}

Introducción: El modelo de datos Simple Knowledge Organization System (SKOS) es una recomendación de World Wide Web Consortium (W3C) diseñada para la representación de los Sistemas de Organización del Conocimiento en entornos digitales. En la última década, el modelo SKOS ha contribuido a un aumento exponencial en la cantidad de vocabularios publicados en entornos digitales, destacando la necesidad de estudios que favorezcan una mejor evaluación de estos vocabularios. Objetivo: Presentar una sistematización de lineamientos para la evaluación de vocabularios publicados en SKOS. Metodología: Investigación exploratoria de carácter bibliográfico y documental, con enfoque teórico y cualitativo. Resultados: Se presenta estudios que pueden contribuir a una sistematización de pautas para la evaluación de vocabularios codificados en SKOS. Conclusiones: la representación de vocabularios controlados en entornos digitales incorpora muchas potencialidades a los sistemas de organización del conocimiento, pero también presenta nuevos desafíos, especialmente en relación con la evaluación de los requisitos técnicos y la definición formal de relaciones, destacando la importancia de los estudios que contribuyen a un mayor refinamiento. Procesos de evaluación de vocabulario en entornos digitales.

Descriptores: Knowledge Organization Systems. Vocabularios Controlados. Evaluación. Directrices. SKOS. 